\title{
LA OPACIFICACIÓN CAPSULAR POSTERIOR: UN FACTOR A TENER EN CUENTA EN LA VALORACIÓN DEL NERVIO ÓPTICO
}

\section{POSTERIOR CAPSULAR OPACIFICATION: ONE FACTOR TO BE CONSIDERED FOR THE STUDY OF THE OPTIC NERVE}

\author{
GARCÍA-MEDINA JJ ${ }^{1}$, GARCÍA-MEDINA M², GONZÁLEZ-OCAMPO-DORTA S ${ }^{3}$
}

Como es sabido la pseudofaquia es una condición muy frecuente en el conjunto de los pacientes oftalmológicos. El beneficio visual obtenido tras la operación de cataratas puede decrecer con el tiempo debido a la aparición progresiva de una opacificación capsular posterior (OCP). A pesar de las continuas modificaciones en las técnicas quirúrgicas y en los diseños y materiales de las lentes intraoculares implantadas, la OCP sigue siendo la complicación postquirúrgica más frecuente a largo plazo en la cirugía de la catarata (1). Las tasas de OCP varían según los estudios pero un meta-análisis publicado en 1998 reportó que globalmente el $25 \%$ de los pacientes que eran sometidos a cirugía de la catarata desarrollaban OCP a los 5 años de la operación (2).

La OCP es secundaria a la proliferación y la migración centrípeta de células cristalinianas epiteliales remanentes del ecuador y del saco capsular anterior hasta llegar a bloquear el eje visual. De acuerdo con los patrones de crecimiento de estas células la opacidad resultante adopta un aspecto fibroso, perlado o, lo que es más frecuente, una combinación de ambos (3).

Dichos cambios en la cápsula posterior pueden conllevar pérdida de agudeza visual, alteración de la sensibilidad al contraste, glare y diplopia monocular $(4,5)$. La metaplasia de células epiteliales causa también complicaciones indirectas relacionadas con tracciones mecánicas. Así, las células epiteliales pueden transformarse en fibroblastos que contienen elementos contráctiles (miofibroblastos) y que causan que la cápsula posterior se arrugue. Estos pliegues incluso ocasionan distorsiones visuales como glare o similares a los ocasionados por una varilla de Maddox (6).

Todas estas formaciones irregulares combinadas de fibrosis, perlas y estrías de la cápsula posterior alteran la reflexión, refracción, difracción y dispersión de la luz en el ojo pudiendo interferir no sólo con la visión del paciente sino también con las pruebas necesarias para examinar el nervio óptico.

En la actividad diaria no es raro tener que valorar en la consulta ojos pseudofáquicos con OCP que puedan tener o que tengan alguna patología concomitante del nervio óptico, como puede ser un glaucoma o alguna enfermedad neuro-oftalmológica. A raíz de situaciones clínicas concretas nos planteamos cómo podría influir la OCP en los resultados de las distintas pruebas que se utilizan para evidenciar el estado funcional y morfológico del nervio óptico y de la capa de fibras nerviosas retinianas peripapilares (CFNRP). Con este fin diseñamos una serie de estudios, comparando los resultados de diferentes pruebas diagnósticas (perimetría automatizada, polarimetría láser y tomografía óptica de

\footnotetext{
${ }^{1}$ Doctor en Medicina. Servicio de Oftalmología. Hospital La Inmaculada. Huercal Overa. Almería; Unidad de Investigación Oftalmológica Santiago Grisola, Hospital Universitario Doctor Peset, Valencia. España.

2 Doctor en Medicina. Servicio de Oftalmología. Hospital Torrecárdenas. Almería. España.

${ }^{3}$ Licenciado en Medicina. Servicio de Oftalmología. Hospital Universitario de Albacete. Albacete. España.
}

Correspondencia:

José Javier García-Medina

Servicio de Oftalmología. Hospital La Inmaculada

Avda. Ana Parra, s/n

04600 Huercal Overa (Almería)

España

E-mail: josegarciam@yahoo.com 
coherencia) antes y después de la realización de una capsulotomía en pacientes afectos de OCP.

Respecto a la perimetría automatizada (PA) observamos que no sólo la desviación media (indicador del grado de depresión global del campo) sino también la desviación estándar de la media (indicador del grado de depresión focal) mejoraron tras la capsulotomía. Este hallazgo confirma que la OCP se comporta como una opacificación heterogénea, es decir, deprime el campo visual más «por zonas» y no tan homogéneamente como lo hacen las cataratas. La idea es compatible con la clínica diaria. El lector habrá podido evidenciar en la lámpara hendidura qué caprichosas formas y texturas adoptan las OCP en distintos pacientes. Por otro lado evidenciamos también que algunos campos previos a la capsulotomía presentaban defectos que simulaban escotomas arqueados. Una vez realizado el láser, dichos artefactos desaparecieron (7). A efectos prácticos, por tanto, se podría confundir en determinados casos un defecto campimétrico producido por una OCP con un defecto campimétrico glaucomatoso (falso positivo).

Estudiando la polarimetría láser (PL) aparecieron también hallazgos interesantes. Encontramos que los parámetros de estimación del grosor de la CFNRP obtenidos mediante GDx-VCC eran significativamente menores tras la capsulotomía, es decir, que existe una sobreestimación de los grosores en pacientes afectos de OCP, asociada con una infraestimación del Nerve Fiber Indicator $(8,9)$. Por tanto, se podría pasar por alto el diagnóstico de glaucoma en ojos con OCP atendiendo a los resultados de la PL (falsos negativos). Por otra parte evidenciamos que la capsulotomía cambiaba la birrefringencia del segmento anterior (BSA), haciéndose necesaria una nueva estimación de la BSA para los sucesivos exámenes con GDx-VCC tras la capsulotomía $(10,11)$.

En cuanto a la tomografía de coherencia óptica (OCT), obtuvimos un aumento significativo de la calidad de imagen (medida en términos de «signal strength» o fuerza de señal) y del número de pruebas valorables tras eliminar la OCP. Sin embargo no encontramos cambios en los grosores de la CFNRP tras la capsulotomía (12). Tal diferencia de resultados entre OCT y PL puede ser debida al diferente funcionamiento de ambos dispositivos (13).

Por todo lo dicho anteriormente, parece recomendable en ojos pseudofáquicos realizar siempre un examen detallado del segmento anterior con lámpara de hendidura para determinar una posible OCP a la hora de interpretar en su justa medida las distintas pruebas que valoran el nervio óptico (PA, PL y OCT). Si se decide realizar una capsulotomía creemos necesario repetir las pruebas tras el láser pues así, una vez eliminados los artefactos ocasionados por la OCP, los valores reales servirán de referencia para hacer comparaciones en el futuro y poder constatar una posible progresión de la patología estudiada (14). Si se prefiere no realizar aún la capsulotomía el método diagnóstico más fiable para el estudio del nervio óptico parece ser la OCT.

\section{BIBLIOGRAFÍA}

1. Findl O, Buehl W, Bauer P, Sycha T. Interventions for preventing posterior capsule opacification. Cochrane Database Syst Rev 2007; 18: CD003738.

2. Schaumberg DA, Dana MR, Christen WG, Glynn RJ. A systematic overview of the incidence of posterior capsule opacification. Ophthalmology 1998; 105: 1213-1221.

3. Apple DJ, Solomon KD, Tetz MR, Assia EI, Holland EY, Legler UF, et al. Posterior capsule opacification. Surv Ophthalmol 1992; 37: 73-116.

4. Meacock WR, Spalton DJ, Boyce J, Marshall J. The effect of posterior capsule opacification on visual function. Invest Ophthalmol Vis Sci 2003; 44: 4665-4669.

5 García Medina JJ, García Medina M, Pinazo Durán MD, Suarez-Varela MM. Monocular diplopia after neodymium: YAG laser capsulotomy. Graefes Arch Clin Exp Ophthalmol 2005; 243: 1288-1290.

6. Holladay JT, Bishop JE, Lewis JW. Diagnosis and treatment of mysterious light streaks seen by patients following extracapsular cataract extraction. J Am Intraocul Implant Soc 1985; 11: 21-23.

7. García-Medina JJ, García-Medina M, Arbona-Nadal MT, Pinazo-Duran MD. Effect of posterior capsular opacification removal on automated perimetry. Eye 2006; 20: 537 545 .

8. Garcia-Medina JJ, Garcia-Medina M, Shahin M, PinazoDuran MD. Posterior capsular opacification affects scanning laser polarimetry examination. Graefes Arch Clin Exp Ophthalmol 2006; 244: 520-523.

9. García-Medina JJ, García-Medina M, Dorta SG, PinazoDurán MD, Gallego-Pinazo R, Zanón-Moreno VC. Effect of posterior capsular opacification removal on scanning laser polarimetry measurements. Graefes Arch Clin Exp Ophthalmol 2006; 244: 1398-1405.

10. Garcia-Medina JJ, Garcia-Medina M, Gonzalez-OcampoDorta S, Gallego-Pinazo R, Scalerandi G, Zanon-Moreno $V C$, Pons-Vazquez S. Anterior Segment Birefringence before and after Nd:YAG Laser Capsulotomy for Posterior Capsule Opacification. Presentación en Poster. Congress of the American Academy of Ophthalmology. Nueva Orleans, Louisiana; 2007.

11. Garcia-Medina JJ, Garcia-Medina M, Zanon-Moreno VC, Gallego-Pinazo R, Pons-Vazquez S, Gonzalez-Ocampo S, et al. The influence of posterior capsular opacification removal on anterior segment birefringence parameters as 
measured by scanning laser polarimetry. Clin Experiment Ophthalmol 2007; 35: 414-420.

12. Garcia-Medina JJ, Gonzalez-Ocampo-Dorta S, FelicianoSanchez A, Scalerandi G, Gallego-Pinazo R, GarciaMedina M. Changes in Optical Coherence Tomography after Posterior Capsular Opacification Removal. Congress of the American Academy of Ophthalmology. Nueva Orleans, Louisiana; 2007.
13. Choplin NT. Scanning laser imaging devices: what do we measure? Clin Experiment Ophthalmol 2007; 35: 401-402.

14. Garcia-Medina JJ, Garcia-Medina M. The Influence of Posterior Capsular Opacification on the Assessment of the Optic Nerve. In: Lewis BJ, Davies CJ. Optic Nerve Disease Research Perspectives. New York: Nova Publishers; 2008. 